\title{
IMMUNOGENICITY AND SAFETY OF INACTIVATED SARS-COV-2 VACCINE (VERO CELL), BBIBP-CORV (SINOPHARM); AN OBSERVATIONAL STUDY FROM PAKISTAN
}

\author{
Muhammad Farooq, Muhammad Fayyaz Malik, Ashfaq Hussain, Majid Latif, Muhammad Usman Rathore, Rehan Ahmad Khan Lodhi
}

Combined Military Hospital Sialkot/National University of Medical Sciences (NUMS) Pakistan

\section{ABSTRACT}

Objective: To ascertain the immunogenicity and short-term safety of inactivated SARS-CoV-2 Vaccine (Vero Cell), BBIBPCorV (Sinopharm) in our setup.

Study Design: Cross-sectional study.

Place and Duration of Study: Combined Military Hospital Sialkot Pakistan, from Feb to Apr 2021.

Methodology: A total of 227 health care workers (HCWs) between 18 to 59 years of age were included in the study. Two doses of Inactivated SARS-CoV-2 Vaccine (Vero Cell), BBIBP-CorV were administered to all individuals 21 days apart and they were monitored for any vaccine-related adverse reactions for 7 days after each dose. Neutralizing antibodies (NAbs) in study subjects were detected in three samples i.e. before 1st dose of vaccine, 21 days after 1st dose and 14 days after 2 nd dose by Elecsys Anti- SARS-CoV-2 S (Roche Diagnostics).

Results: Mean age of individuals in the study was $36.70 \pm 18.08$ years and most individuals were in the 31-45 years age group. Fatigue and drowsiness were the most common adverse effects experienced by study subjects after 1st and 2nd dose of the vaccine followed by malaise and headache. Only $42(39 \%)$ individuals developed positive neutralizing antibody titers in a sample taken 21 days after 1st dose while all individuals except one (99\%) developed positive neutralizing antibody titers in a sample taken 2 weeks after 2nd vaccine dose.

Conclusion: Inactivated SARS-CoV-2 Vaccine (Vero Cell), BBIBP-CorV is safe and well-tolerated with very few adverse reactions. Immunogenicity was well achieved as the seroconversion rate was $99 \%$ two weeks after 2nd dose of the vaccine.

Keywords: Coronavirus disease 2019 (COVID-19), Inactivated SARS-CoV-2 vaccine, Neutralizing antibodies (NAbs).

How to Cite This Article: Farooq M, Malik MF, Hussain A, Latif M, Rathore MU, Lodhi RAK. Immunogenicity and Safety of Inactivated Sars-Cov-2 Vaccine (Vero Cell), Bbibp-Corv (Sinopharm); An Observational Study from Pakistan. Pak Armed Forces Med J 2021; 71(6): 2024-2028. $\quad$ Doi:https://doi.org/10.51253/pafmj.v6i6.7034

This is an Open Access article distributed under the terms of the Creative Commons Attribution License (https://creativecommons.org/licenses/by-nc/4.0/), which permits unrestricted use, distribution, and reproduction in any medium provided the original work is properly cited.

\section{INTRODUCTION}

COVID-19 caused by severe acute respiratory syndrome- coronavirus 2 (SARS-CoV-2) was declared a pandemic on 11th March 2020 by World Health Organization (WHO). The increase in COVID-19 cases was at a shocking rate throughout the world. ${ }^{1}$ As of 25 May 2021, the number of COVID-19 cases has reached above 166.86 million worldwide with more than 3.45 million deaths. ${ }^{2}$ COVID-19 has caused social and economic instability and it proved to be disastrous to public health placing life on hold worldwide since December 2019. ${ }^{3}$ There is a shortage of medical necessities, hospitals have become overburdened, and health care workers have been drained as a result of the constant fight against the pandemic. ${ }^{4}$ Isolation, physical distancing and wearing of masks were useful in restricting the spread of infection in the short term. Older individuals, those with comorbid conditions and healthcare workers are at significantly high risk. ${ }^{5-6}$

The development of a vaccine is of utmost

Correspondence: Dr Muhammad Farooq, Colsultant Microbiologist Combined Military Hospital Sialkot-Pakistan

Received: 06 Jul 2021; revision received: 17 Sep 2021; accepted: 30 Sep 2021 urgency and importance to curb the global pandemic and precipitously growing numbers of death. ${ }^{1,4}$ Multiple vaccine types against SARS-CoV-2 are under development which includes DNA, RNA based vaccines, recombinant vaccines, vector-based vaccines and inactivated virus vaccines. ${ }^{17-9}$ Moderna and Pfizer/Bio NTech are two important RNA vaccines that encode SARS-CoV-2 spike glycoprotein, Novavax a protein subunit vaccine while vaccines of Astra Zeneca (Oxford University), CanSino, Janssen/Johnson \& Johnson and Gamaleya comprise adenovirus vector platforms expressing spike protein. 3,4,9-10 There are several Chinese vaccines in development while Sinovac and Sinopharm have received emergency use approval in China and various other countries. These two vaccines are comprised of the whole inactivated virus where alum (Aluminium hydroxide) is an adjuvant. $5,8,10$ Sinopharm has now announced vaccine efficacy of $79 \% .{ }^{10}$ Inactivated vaccines have extensively been used to control the spread of various infectious diseases and these types of vaccines are developed at a relatively fast speed which makes them an encouraging strategy for vaccine development against COVID-19.7 Viruses are grown in cell culture of mammalian cells and then 
inactivated with either $\beta$-propiolactone or formaldehyde. ${ }^{3}$ The safety and high immunogenicity is pivotal for vaccine deve-lopment to control the COVID-19 pandemic and pre-vent further fatalities. ${ }^{7}$

The rationale of this study was to observe adverse reactions to inactivated SARS-CoV-2 vaccine (Vero Cell) and the development of neutralizing antibodies in our population which will help us to know the immunogenicity of the vaccine and allow us to find out the safety of the vaccine to prevent unnecessary fears related to vaccine among general populations.

\section{METHODOLOGY}

This cross-sectional study was conducted in the Microbiology Department, Combined Military Hospital Sialkot, from February to April 2021. Informed consent was taken from all the participants and the study was approved by institutional review board (certificate no. ERC/05/2021). The sample size was calculated by the World Health Organization (WHO) sample size calculator using the prevalence of COVID-19 as 7.2\%. ${ }^{11}$

Inclusion Criteria: Health care workers (HCWs) i.e. medical doctors, nursing staff and helpers in various wards and intensive care units, between 18 to 59 years of age were included in the study.

Exclusion Criteria: Health care workers who were immune-deficient or who were on some immunosuppressive drugs were excluded from the study. Pregnant and lactating mothers were also excluded from the study.

These HCWs were selected by non-probability consecutive sampling technique in the study. The vaccine was stored and transported at $2-8^{\circ} \mathrm{C}$ and it was protected from light as per manufacturer's guidelines. The safety and immunogenicity of vaccines were determined in our study. Safety was determined by finding out adverse reactions (local adverse effects like injection site pain and swelling etc and systemic side effects like fever, fatigue, malaise, headache and drowsiness etc) within 7 days of each vaccine dose while immunogenicity was ascertained by the response of neutralizing antibodies against SARS-CoV-2.

Blood sample of all participants of the study was taken before 1st dose of vaccine for neutralizing antibody titers (1st Sample) and they were given 1st dose of $0.5 \mathrm{ml}$ inactivated SARS-CoV-2 vaccine (Vero Cell) through intramuscular route into the deltoid muscle of the non-dominant side. All of them were observed for 30 minutes for any immediate side effects and allergic reactions. They were asked to report again if they develop any symptoms within 7 days of 1 st dose. Then they had to report for 2nd dose of intramuscular vaccine 21 days after the 1st dose. On the 21st day, before administering 2 nd dose of vaccine, a blood sample was taken again (2nd sample) for neutralizing antibodies and 2nd dose of vaccine was given. The same protocol of observation for 30 minutes was followed and participants were asked to report again in case of development of any symptoms within 7 days of 2nd dose. They were sent home and asked to report back for 3rd sample of blood 14 days after 2 nd dose of the vaccine. Neutralizing antibodies in all three samples were detected by Elecsys Anti-SARS-CoV-2S (Roche Diagnostics) run on Roche's Cobas e411 analyzer (Electro Chemi Luminescence based immunoassay system). This test was used to determine antibodies against spike proteins in human serum and plasma quantitatively. Titers of Elecsys Anti- SARS-CoV-2 S $\geq 0.8 \mathrm{U} / \mathrm{ml}$ were considered positive while titers $<0.8 \mathrm{U} / \mathrm{ml}$ were considered negative.

The data was entered and analyzed by Statistical Package for the social sciences (SPSS) version 23. Descriptive statistics were calculated for both qualitative and quantitative variables. For quantitative variables like age and neutralizing antibody titers, mean and standard deviation (SD) were calculated. For qualitative variables like gender, frequency and percentage were calculated.

\section{RESULTS}

A total of 227 individuals were included in the study, all of them were followed for vaccine safety and development of any side effects but only 107 individuals $(47 \%)$ were followed for immune response. Rest 120 individuals (53\%) who were not followed for immunogenicity included 25 people ( $11 \%$ ) who already had developed COVID-19 before vaccination, 90 individuals $(40 \%)$ who had significant neutralizing antibody titers in their 1st sample taken before 1st dose of vaccine although they had no known history of previous COVID-19 and 5 people (2\%) who developed COVID19 during the study period. The mean age of individuals in the study was $36.70 \pm 18.08$ years while the minimum age was 21 years and the maximum age was 57 years. Most individuals were in the 31-45 years age group. Male study participants were more than female participants. Fatigue and drowsiness were the most common adverse effects experienced by study subjects after 1st and 2nd dose of the vaccine followed by malaise and headache. Details of adverse effects have been mentioned in Table-I. Different ranges of quantitative 
antibody titers with relation to a number of individuals have been mentioned in Table II.

Table-I: Characteristics of study participants and adverse effects experienced by them.

\begin{tabular}{|c|c|}
\hline Characteristics & n (\%) \\
\hline \multicolumn{2}{|l|}{ Gender } \\
\hline Male & $125(55)$ \\
\hline Female & $102(45)$ \\
\hline \multicolumn{2}{|l|}{ Age Groups (Years) } \\
\hline $18-30$ & $72(32)$ \\
\hline $31-45$ & $103(45)$ \\
\hline$>45$ & $52(23)$ \\
\hline \multicolumn{2}{|l|}{ Adverse Effects } \\
\hline Injection site pain & $10(4.4)$ \\
\hline Injection site swelling & $2(0.9)$ \\
\hline Injection site redness & $2(0.9)$ \\
\hline Fever & $6(2.6)$ \\
\hline Headache & $13(5.7)$ \\
\hline Fatigue & $22(9.7)$ \\
\hline Muscle pain & $3(1.3)$ \\
\hline Chills & - \\
\hline Joint pain & - \\
\hline Malaise & $11(4.8)$ \\
\hline Lymphadenopathy & - \\
\hline Diarrhoea & $4(1.7)$ \\
\hline Nausea/ vomiting & $3(1.3)$ \\
\hline Drowsiness & $21(9)$ \\
\hline Dizziness & $8(3.5)$ \\
\hline Syncope & $1(0.4)$ \\
\hline No adverse effects & $171(75)$ \\
\hline
\end{tabular}

Table-II: Neutralizing antibody titers with respect to number of individuals $(n=107)$.

\begin{tabular}{l|c|c|c}
\hline Titers & $\begin{array}{c}\mathbf{1}^{\text {st }} \text { Sample } \\
\mathbf{n}(\mathbf{\%})\end{array}$ & $\begin{array}{c}\mathbf{2}^{\text {nd }} \text { Sample } \\
\mathbf{n} \mathbf{( \% )}\end{array}$ & $\begin{array}{c}3^{\text {rd }} \text { Sample } \\
\mathbf{n}(\mathbf{\%})\end{array}$ \\
\hline Negative & $107(100)$ & $65(61)$ & $01(1)$ \\
\hline Positive & - & $42(39)$ & $106(99)$ \\
\hline$<10(\mathrm{U} / \mathrm{ml})$ & - & $35(33)$ & $16(15)$ \\
\hline $10-20(\mathrm{U} / \mathrm{ml})$ & - & $3(3)$ & $10(9)$ \\
\hline $21-100(\mathrm{U} / \mathrm{ml})$ & - & $2(2)$ & $34(33)$ \\
\hline$>100(\mathrm{U} / \mathrm{ml})$ & - & $2(2)$ & $16(15)$ \\
\hline$\geq 250(\mathrm{U} / \mathrm{ml})$ & - & - & $30(29)$ \\
\hline
\end{tabular}

All 7 individuals with titers $>10$ in the 2 nd sample developed titers of more than 250 in the 3rd sample. Ranges of antibody titers in the 3rd sample which would determine immunogenicity (taken 2 weeks after 2nd vaccine dose) with relation to gender and different age groups have been shown in Table-III. Only 1 male of 47 years of age did not develop reactive titers of neutralizing antibodies in the 3rd sample (Table-IV).

\section{DISCUSSION}

Vaccination has become undoubtedly relevant as one of the major contributions which modern medicine can offer for the long term prevention of ongoing pandemics. Keeping in mind the significance of the vac- cine in fighting this public health emergency, understanding the allergic reactions and other side effects of vaccines is crucial.,12-14 Process of vaccine development comprises of different stages which include preclinical trials which assess safety and immunogenicity in animal models or cell culture. Clinical trials are conducted in human subjects which include phase 1, 2 and 3 vaccine trials. ${ }^{15-18}$ High immunogenicity and efficacy of vaccines are equally important to prevent further spread of cases. ${ }^{7}$ As per Sinopharm interim analysis of phase 3 trials in UAE review, its efficacy was found to be $86 \%$ against COVID-19 infection and effectiveness was $100 \%$ in preventing moderate and severe disease, 3,13 while as per Sinopharm claim based on interim phase 3 trials data it was $79 \%$ effective against symptomatic COVID-19 prevention. ${ }^{13,19}$

Table-III: Neutralizing antibody titers in 3rd Sample with respect to gender of individuals.

\begin{tabular}{l|c|c}
\hline Titers & Male, $\mathbf{n}(\mathbf{\%})$ & Female, $\mathbf{n}(\mathbf{\%})$ \\
\hline & $\mathrm{n}=63$ & $\mathrm{n}=44$ \\
\hline Negative & $1(1.6)$ & - \\
\hline Positive & $62(98.4)$ & $44(100)$ \\
\hline$<10(\mathrm{U} / \mathrm{ml})$ & $8(12.7)$ & $8(18.2)$ \\
\hline $10-20(\mathrm{U} / \mathrm{ml})$ & $5(7.9)$ & $5(11.4)$ \\
\hline $21-100(\mathrm{U} / \mathrm{ml})$ & $26(41.3)$ & $8(18.2)$ \\
\hline$>100(\mathrm{U} / \mathrm{ml})$ & $10(15.9)$ & $6(13.6)$ \\
\hline$\geq 250(\mathrm{U} / \mathrm{ml})$ & $13(20.6)$ & $17(38.6)$ \\
\hline
\end{tabular}

Table-IV: Neutralizing antibody titers in 3rd Sample with respect to age groups of individuals.

\begin{tabular}{l|c|c|c}
\hline Titers & $\begin{array}{c}\mathbf{1 8 - 3 0} \text { years } \\
\mathbf{n}(\mathbf{\%})\end{array}$ & $\begin{array}{c}\mathbf{3 1 - 4 5} \text { years } \\
\mathbf{n}(\mathbf{\%})\end{array}$ & $\begin{array}{c}\mathbf{> 4 5} \text { years } \\
\mathbf{n}(\mathbf{0})\end{array}$ \\
\hline & $\mathrm{n}=32$ & $\mathrm{n}=45$ & $\mathrm{n}=30$ \\
\hline Negative & - & - & $1(3.3)$ \\
\hline Positive & $32(100)$ & $45(100)$ & $29(96.7)$ \\
\hline$<10(\mathrm{U} / \mathrm{ml})$ & $5(15.6)$ & $3(6.7)$ & $8(26.7)$ \\
\hline $10-20(\mathrm{U} / \mathrm{ml})$ & $2(6.3)$ & $8(17.8)$ & - \\
\hline $21-100(\mathrm{U} / \mathrm{ml})$ & $18(56.3)$ & $11(24.4)$ & $5(16.7)$ \\
\hline$>100(\mathrm{U} / \mathrm{ml})$ & - & $13(28.9)$ & $3(10)$ \\
\hline$\geq 250(\mathrm{U} / \mathrm{ml})$ & $7(21.9)$ & $10(22.2)$ & $13(43.3)$ \\
\hline
\end{tabular}

In our study, we included only health care workers (HCWs) of 18-59 years of age. All study participants were given a vaccine dose and none of them received a placebo. Fatigue and drowsiness were the most predominant adverse reactions found in our study subjects followed by malaise and headache within 7 days of vaccine doses. All study participants developed mild to moderate adverse reactions and none of them experienced severe adverse effects. These findings were comparable to the Phase $1 / 2$ trial of BBIBPCorV vaccine conducted by Xia et al, ${ }^{20}$ who concluded that within 28 days after vaccination no severe adverse reactions were reported and the vaccine was considered well-tolerated and safe in all doses but contrary to 
our study most common systemic adverse effect in that trial was fever. The incidence of local injection site pain was also lower in our study (4.4\%). Interim data of phase 3 trials also reported that there were no severe safety concerns. ${ }^{3}$ Another phase 1/2 trials conducted on an inactivated vaccine, CoronaVac (Sinovac) showed similar results. Most of the adverse effects experienced by study subjects were mild in that study as well. ${ }^{5}$ Studies conducted on the safety of the BNT162b2 mRNA Vaccine showed that the incidence of severe adverse reactions was less and was similar in placebo and vaccine groups. ${ }^{6,21}$ Another study conducted by Zhu et al, on the safety of adenovirus type- 5 vectored vaccine showed a higher incidence of adverse reactions as compared to our study but most adverse reactions reported by them were mild to moderate..$^{22}$

In our study mean neutralizing antibody titers in the 3rd sample (14 days after 2 nd dose) were way higher than the 2 nd sample (21 days after the 1st dose). Only 42 (39\%) individuals developed positive NAbs titers in the 2nd sample, out of which 35 individuals had titers $<10 \mathrm{U} / \mathrm{ml}$ while in the 3rd sample all individuals except one $(99 \%)$ developed positive NAbs titers and only 16 individuals had titers $<10 \mathrm{U} / \mathrm{ml}$. Our findings were comparable to another study conducted by Xia et al, in which the seroconversion rate was found to be $100 \%$ which was achieved earlier in the younger age group of 18-59 years as compared to the 60 years and older group.., 20 Our findings were also comparable to the interim analysis of the phase 3 trial in which the NAbs seroconversion rate was found to be $99 \% .^{3} \mathrm{Imm}-$ unogenicity was achieved slightly better in our study as compared to studies conducted on CoronaVac (Sinovac). ${ }^{3,5}$

In a trial conducted by Xia et al. Neutralizing antibody titers magnitude was lower in 60 years and older age group as compared to the age group of 18-59 years. ${ }^{20}$ There was no significant difference of NAbs titers in different age groups considered in our study. Only one individual who did not develop reactive titers of neutralizing antibodies was of 47 years of age. This was most likely because all age groups considered in our study were between 18-59 years of age and there were no individuals more than 60 years of age.

\section{LIMITATION OF THE STUDY}

There were certain limitations in our study. The study was only conducted on individuals of 18-59 years of age so safety and immunogenicity in the older population who are more at risk cannot be ascertained. There was only short term follow-up for adverse reactions to the vaccine. This was an observational study in which there was no placebo group so the correlation of adverse reactions with the vaccine cannot be confirmed. There was 3rd wave of COVID-19 going on in the country during the study period and the study included only health care workers who were significantly exposed to SARS-CoV-2 so it was difficult to determine if NAbs titers were reached because of actual exposure or because of the vaccine.

\section{CONCLUSION}

We concluded that inactivated SARS-CoV-2 Vaccine (Vero Cell), BBIBP-CorV is safe, well-tolerated with adverse reactions of only mild to moderate severity. Seroconversion rate was $99 \%$ at 14 days after 2 nd dose while it was just 39\% 21 days after 1st dose of the vaccine so significant humoral response against SARS-CoV-2 was induced after two doses. There is a need for further investigations on this vaccine for control of COVID-19 and its efficacy remains to be ascertained.

\section{CONFLICT OF INTEREST}

The authors have no financial or other interests with regard to the submitted manuscript that might be construed as a conflict of interest.

\section{Conflict of Interest: None.}

\section{Authors' Contribution}

MF: Conception, design analysis \& interpretation of data, MFM: Conception, design \& write up, AH: Design, analysis \& interpretation of data, ML: Analysis \& interpretation of data, MUR: interpretation of data \& proofreading, RAKL: Interpretation of data, proofreading.

\section{REFERENCES}

1. Gao Q, Bao L, Mao H, Wang L, Xu K, Yang M, et al. Development of an inactivated vaccine candidate for SARS-CoV-2. Sci 2020; 369(6499): 77-81.

2. World Health Organization. Coronavirus Disease (COVID-19) Dashboard. [Internet] Available at: https://www.covid19.who. int [Accessed on May 25, 2021].

3. Ophinni Y, Hasibuan AS, Widhani A, Maria S, Koesnoe S, Yunihastuti E, et al. COVID-19 Vaccines: current status and implication for use in Indonesia. Acta Med Indones 2020; 52(4): 388-392.

4. Banerji A, Wickner PG, Saff R, Stone Jr CA, Robinson LB, Long AA, et al. mRNA vaccines to prevent COVID-19 disease and reported allergic reactions: current evidence and approach. J Allergy Clin Immunol Pract 2020; 9(4): 1423-1437.

5. Zhang Y, Zeng G, Pan H, Li C, Hu Y, Chu K, et al. Safety, tolerability, and immunogenicity of an inactivated SARS-CoV-2 vaccine in healthy adults aged 18-59 years: a randomized, doubleblind, placebo-controlled, phase $1 / 2$ clinical trial. Lancet Infect Dis 2021; 21(2): 181-192.

6. Polack FP, Thomas SJ, Kitchin N, Absalon J, Gurtman A, Lockhart S, et al. Safety and efficacy of the BNT162b2 mRNA COVID-19 vaccine. N Engl J Med 2020; 383(27): 26032615.

7. Wang H, Zhang Y, Huang B, Deng W, Quan Y, Wang W, et al. Development of an inactivated vaccine candidate, BBIBP-CorV, with potent protection against SARS-CoV-2. Cell 2020; 182(3): 713-721.

8. Krammer F. SARS-CoV-2 vaccines in development. Nature 2020; 586(7830): 516-527. 
9. Dong Y, Dai T, Wei Y, Zhang L, Zheng M, Zhou F. A systematic review of SARS-CoV-2 vaccine candidates. Signal Transduct Target Ther 2020; 5(1): 1-4.

10. Kim JH, Marks F, Clemens JD. Looking beyond COVID-19 vaccine phase 3 trials. Nat Med 2021; 27(2): 205-211.

11. Pakistan COVID-19 Situation Report, December 2020. [Internet] Available at: https://www.unicef.org/documents/pakistancovid-19-situation-report-december-2020 [Accessed on Sep 16, 2021].

12. Awadasseid A, Wu Y, Tanaka Y, Zhang W. Current advances in the development of SARS-CoV-2 vaccines. Int J Biol Sci 2021; 17(1): 8-12.

13. Baraniuk $C$. What do we know about China's covid-19 vaccines? BMJ 2021; 373(1): n912.

14. Xia S, Duan K, Zhang Y, Zhao D, Zhang H, Xie Z, et al. Effect of an inactivated vaccine against SARS-CoV-2 on safety and immunogenicity outcomes: interim analysis of 2 randomized clinical trials. JAMA 2020; 324(10): 951-960.

15. Plotkin S, Robinson JM, Cunningham G, Iqbal R, Larsen S. The complexity and cost of vaccine manufacturing-an overview. Vaccine 2017; 35(33): 4064-4071.

16. Van-Doremalen N, Lambe T, Spencer A, Belij-Rammerstorfer S, Purushotham JN, Port JR, et al. ChAdOx1 nCoV-19 vaccine prevents SARS-CoV-2 pneumonia in rhesus macaques. Nature 2020; 586(7830): 578-582.
17. Yu J, Tostanoski LH, Peter L, Mercado NB, McMahan K, Mahrokhian SH, et al. DNA vaccine protection against SARSCoV-2 in rhesus macaques. Sci 2020; 369(6505): 806-811.

18. Corbett KS, Flynn B, Foulds KE, Francica JR, Boyoglu-Barnum $S$, Werner AP, et al. Evaluation of the mRNA-1273 vaccine against SARS-CoV-2 in nonhuman primates. N Engl J Med 2020; 383(16) 1544-1555.

19. Reuters. Sinopharm's COVID-19 vaccine $79 \%$ effective, seeks approval in China. [Internet] Available at: https://www. reuters.com/article/us-health-coronavirus-chinavaccine/ sinopharms-covid-19-vaccine-79-effective-seeksapproval-in-china-idUSKBN2940C8 [Accessed on May 27, 2021].

20. Xia S, Zhang Y, Wang Y, Wang H, Yang Y, Gao GF, et al. Safety and immunogenicity of an inactivated SARS-CoV-2 vaccine, BBIBP-CorV: a randomised, double-blind, placebo-controlled, phase 1/2 trial. Lancet Infect Dis 2021; 21(1): 39-51.

21. Walsh EE, Frenck Jr RW, Falsey AR, Kitchin N, Absalon J, Gurtman A, et al. Safety and immunogenicity of two RNA-based Covid-19 vaccine candidates. New Engl J Med 2020; 383(25): 2439-2450.

22. Zhu FC, Li YH, Guan XH, Hou LH, Wang WJ, Li JX, et al. Safety, tolerability, and immunogenicity of a recombinant adenovirus type- 5 vectored COVID-19 vaccine: a dose-escalation, open-label, non-randomised, first-in-human trial. Lancet 2020; 395(10240): 1845-1854 